\title{
Citoyenneté européenne, transnationale ou globale : penser la citoyenneté au-delà de l'État. Analyse critique d'un débat
}

\author{
Élise Auvachez
}

Résumé

Objet politique pas toujours identifié, mais souvent controversé, la citoyenneté européenne, près de 15 ans après son introduction dans le Traité de Maastricht, continue de susciter le débat : quelle citoyenneté pour quelle Europe ? Quelle autonomie pour une citoyenneté européenne dont l'acquisition reste soumise à des règles nationales ? Finalement, la citoyenneté européenne est-elle possible ?

La présente communication propose de lire la problématique de la citoyenneté européenne à la lumière d'un prisme élargissant l'intégrant dans un questionnement dépassant le débat spécifiquement européen, celui de la gouvernance supranationale. Les dynamiques de globalisation et de fragmentation qui caractérisent le contexte contemporain se traduisent dans la sphère politique par la multiplication des paliers de régulation. La sphère nationale n'est plus dans cette perspective le seul locus de la vie politique ; les enjeux et les rapports de force politiques se jouent à des échelles multiples. Ce constat a donné naissance à une prolifération d'analyses concernant la dimension de plus en plus européenne, post/trans/supra-nationale, voire globale, de la citoyenneté. Celles-ci constituent néanmoins un corpus théorique un corpus théorique marqué par une compréhension à la fois fractionnée et diluée de la notion de citoyenneté supranationale et caractérisé par une perspective statique conférant au débat entre les partisans d'une citoyenneté au-delà de l'État et les avocats d'une citoyenneté par essence nationale une circularité stérile. La démarche théorique proposée consiste alors moins à tenter de caractériser les changements contemporains à la lumière des critères de la citoyenneté dans une perspective dichotomique pour savoir si « la » citoyenneté est nationale ou postnationale mais plutôt à comprendre la « citoyennisation », la construction et la définition d'une citoyenneté au-delà de l'État-nation, en relation avec la constitution et la consolidation d'entités politiques supranationales. 
Objet politique pas toujours identifié, mais souvent controversé, la citoyenneté européenne, près de 15 ans après son introduction dans le Traité de Maastricht, continue de susciter le débat : quelle citoyenneté pour quelle Europe ? Quelle autonomie pour une citoyenneté européenne dont l'acquisition reste soumise à des règles nationales ? Finalement, la citoyenneté européenne est-elle possible ${ }^{1}$ ?

Le présent article propose de lire la problématique de la citoyenneté européenne à la lumière d'un prisme élargissant l'intégrant dans un questionnement dépassant le débat spécifiquement européen. Car la dernière interrogation formulée ci-dessus en termes européens renvoie à une réflexion plus large sur la possibilité d’une citoyenneté au-delà de l’État. Si l’Union européenne représente indubitablement le cadre de gouvernance supraétatique le plus abouti, son développement mérite d’être placé dans le cadre plus général des transformations contemporaines de la sphère politique. Les dynamiques de globalisation et de fragmentation qui caractérisent le contexte contemporain se traduisent dans la sphère politique par la multiplication des paliers de régulation. La superposition, ou plutôt l'enchevêtrement, des institutions nationales, des entités infra-étatiques (problématique de la décentralisation) et des organisations supranationales (à caractère régional et international) caractérise cette nouvelle structure de gouvernance, une gouvernance «multi-niveaux », au sein de laquelle l'État, tout en conservant un rôle privilégié, ne détient plus le monopole de la gestion des affaires publiques. La sphère nationale n'est plus dans cette perspective le seul locus de la vie politique ; les enjeux et les rapports de force politiques se jouent à des échelles multiples. Ce constat a donné naissance à une prolifération d'analyses concernant la dimension de plus en plus

\footnotetext{
${ }^{1}$ D’après le titre de l'article de Étienne Balibar (1992).
} 
européenne, post/trans/supra-nationale, voire globale, de la citoyenneté ${ }^{2}$; selon ces travaux, le locus de la citoyenneté est de moins en moins national et de plus en plus supranational. Cet argument commun s'accompagne toutefois d'une multiplicité de définitions associées au concept de citoyenneté; mais en dépit de ces multiples angles d’analyse, les travaux contemporains sur la citoyenneté au-delà de l’État partagent une conception exclusive et une approche réifiante de la citoyenneté. Il en résulte un corpus théorique marqué par une compréhension à la fois fractionnée et diluée de la notion de citoyenneté supranationale et caractérisé par une perspective statique conférant au débat entre les partisans d'une citoyenneté au-delà de l’État et les avocats d’une citoyenneté par essence nationale une circularité stérile. La démarche théorique proposée consiste alors moins à tenter de caractériser les changements contemporains à la lumière des critères de la citoyenneté dans une perspective dichotomique pour savoir si «la » citoyenneté est nationale ou post-nationale mais plutôt à comprendre la «citoyennisation ", la construction et la définition d'une citoyenneté au-delà de l’État-nation, en relation avec la constitution et la consolidation d'entités politiques supranationales.

\section{Une multiplicité de définitions partielles}

\section{Plusieurs conceptualisations différentes de la citoyenneté au-delà de l'État}

La littérature sur la citoyenneté post/trans/supra-nationale n’échappe pas à la critique formulée par plusieurs auteurs à l'égard des analyses sur la citoyenneté dans le cadre national : l’absence d’une théorie intégrée (Tilly 1995a, Turner 1993). Comme les

\footnotetext{
${ }^{2}$ Un champ important de la science politique est également consacré à la citoyenneté urbaine, mais cette perspective infra-étatique n’est pas l'objet de la recherche menée ici.
} 
études autour de la citoyenneté nationale, les analyses sur la citoyenneté post/trans/supranationale sont marquées par une multiplicité de définitions du concept.

L’émergence et le développement exponentiel de systèmes de droits internationaux et régionaux constituent tout d'abord pour plusieurs auteurs la pierre angulaire d'une citoyenneté au-delà de l'État-nation. La base juridique sur laquelle s'est construite l'Union européenne et le système de reconnaissance des droits qui la caractérisent offrent pour plusieurs auteurs l'exemple le plus abouti du développement d’une citoyenneté au-delà de l’État. L’ordre légal distinct sur lequel est fondée l’Union européenne constitue alors le support de l'analyse d'une citoyenneté européenne en gestation (notamment Shaw, 1998 ; Meehan, 1993 et 2000 ; Preuss et al., 2003 ). Elizabeth Meehan montre ainsi comment les droits sociaux développés au sein de l’Union européenne contribuent à la création d’une citoyenneté proprement européenne, même si elle reconnaît que celle-ci présente encore un certain nombre de défauts (1993). Jo Shaw souligne alors la dimension légale historiquement conférée au concept de citoyenneté dans le contexte de l’Union européenne (1998). Tout en reconnaissant que le concept de citoyenneté n’est pas un concept purement légal et qu'il est ancré dans une culture politique, la définition de la citoyenneté en termes récemment privilégiée par Ulrich Preuss et ses collègues dans le numéro spécial de Citizenship Studies consacré à la citoyenneté européenne s’inscrit également dans cette approche juridique : «Citizenship is the status that encompasses the rights, duties, benefits and burdens that follow from a person's nationality» (2003: 7). "Thus, citizenship is defined as the set of (predominantly, but not necessarily legal) rules that constitute a person as a competent member of the polity» (2003: 8). Dans une perspective plus internationale, à partir d'une 
analyse de l'incorporation des travailleurs immigrés dans l'ordre social et institutionnel des différents États européens, Yasemin Soysal $(1994,1997)$ constate l’émergence depuis la Seconde Guerre mondiale d'une nouvelle conception de la citoyenneté : une citoyenneté «post-nationale» universelle, fondée sur la personnalité et non sur l'appartenance nationale, et dont les principes normatifs sont définis au sein des discours et structures transnationaux. Dans la même perspective, Rainer Bauböck (1994) voit dans les droits promus par les organisations transnationales en faveur des migrants et l'acceptation de ces droits par les États le développement d'une conception « transnationale » de la citoyenneté. Enfin, l’acquisition de plus en plus fréquente d’un double, voire triple, passeport par un nombre croissant d'individus constitue pour certains analystes le signe d’une «dé-nationalisation » (Bosniak 1994) croissante de la citoyenneté (Soysal 1997 ; Spiro 1997). Selon Yasemin Soysal, l'existence de doubles citoyennetés, qui remettent en question la notion de loyauté envers un seul état associée à la conception traditionnelle de la citoyenneté, illustre bien l'émergence de formes « postnationales » de la citoyenneté (1997 : 512).

L’ensemble de ces analyses procède d'une conception libérale de la citoyenneté ; celle-ci est associée à un statut légal dont le bénéfice donne accès à un certain nombre de droits. Cette approche en termes de droits renvoie clairement à celle formulée par le sociologue britannique T.H Marshall : «Citizenship is a status bestowed on those who are full members of the community. All who possess the status are equal with respect to the rights and duties with which the status is endowed »(1963: 87). A la lumière de l'histoire de la Grande-Bretagne, T.H Marshall conçoit la citoyenneté à travers un modèle tripartite de droits civils, politiques puis sociaux. 
Envisagée dans une perspective supranationale, l'approche légale de la citoyenneté permet de mettre explicitement en lumière comment une partie importante des droits individuels sont désormais définis et garantis au-delà du cadre étatique par des institutions régionales ou internationales. Considérant la citoyenneté dans une perspective juridique, elle occulte toutefois la dimension politique du concept : celui- renvoie non seulement à un statut légal mais également à une activité politique. Les travaux de Yasemin Soysal montrent ainsi bien comment les principes juridiques de la citoyenneté sont de plus en plus définis au niveau international; son tableau comparatif des citoyennetés nationale et postnational indique cependant que l'organisation de la citoyenneté comme pratique exprimant une appartenance relève toujours de l'Etat (Soysal, 1994: 140). Un tel argument constitue une limite importante à la définition d’une théorie générale de la citoyenneté post-nationale.

L’analyse de la transnationalisation des pratiques politiques est alors au cœur d'un pan important de la littérature sur la citoyenneté supranationale. Un certain nombre d'auteurs voient en effet dans la multiplication des mouvements sociaux transnationaux et l'émergence d'une société civile globale le signe le plus évident de la dénationalisation de la citoyenneté. Richard Falk décrit ainsi l'activisme transnational comme un mode de « citoyenneté globale »; le « réformiste global », avocat du gouvernement mondial et partisan d'une ONU plus forte pour un monde meilleur, est le premier portrait qu'il dresse de ce que signifie être un «citoyen global » (1994 : 132-133). Selon Warren Magnusson : «[T]ransnational social movements involve people in active citizenship and thus lay claim to a political space that may or may not conform to the spaces allowed by 
the existing system of government » (1996 : 9-10). James Tully voit dans la prolifération de réseaux «contre-hégémoniques » reliant organisations et acteurs locaux au sein de réseaux globaux le développement d’une « citoyenneté diverse glocale » (2005 : 13). La société civile globale en émergence résultant de l'agrégation de ces mouvements sociaux transnationaux de plus en plus nombreux constitue pour plusieurs auteurs le principal support de la citoyenneté supranationale (notamment Walzer, 1995 ; Wapner, 1995 ; Lipschutz, 1996 ; Spiro, 1999 : 625-630 ; Anheier et al., 2001 ; Tehranian, 2002 ; Sahle, 2005) : «It is through international civil society that people can best act as global citizens » (Lister, $1997:$ 63).

Fondée sur une conception républicaine de la citoyenneté, exprimée notamment dans les écrits de Rousseau et considérant la condition citoyenne moins en termes de statut qu’en termes de véritable «office » (van Gunsteren, 1994), d'activité politique d'engagement, l'ensemble de cette littérature met clairement en exergue le développement de pratiques de citoyenneté au-delà du cadre stato-national. La société civile globale dont beaucoup des auteurs cités décrivent l'émergence constitue un argument de poids en faveur de l'hypothèse du développement d'une citoyenneté postnationale ; elle ne saurait cependant constituer à elle seule le locus d'une citoyenneté supranationale, et encore moins le point d'ancrage d'une théorie générale de la citoyenneté supranationale. Les travaux associant société civile internationale et citoyenneté post-nationale font de façon générale souvent abstraction des arènes politiques, du cadre institutionnel au sein desquelles s'exerce cette citoyenneté : l'entité politique au sein de laquelle la citoyenneté supranationale émerge est rarement clairement définie, ni même souvent mentionnée. 
La problématique de l'entité politique sur laquelle serait fondée toute forme de citoyenneté postnationale, et par conséquent de l’identité ou de l'appartenance dont celleci serait l'expression, mobilise une partie importante de la littérature. La construction de communautés politiques «post-Westphaliennes» fondées sur le dialogue (« dialogic communities ») et la promotion d’une citoyenneté transnationale constituent ainsi le cœur du «projet critique » proposé par Andrew Linklater (1998 et 2001). Celui-ci s'inspire explicitement dans son ouvrage Transformation of Political Community (1998) des travaux de philosophie politique de Habermas, dont la notion d' «action communicative » (1989 et 1990) rend possible l'existence de communautés politiques supra-étatiques fondées sur le dialogue et la communication. Habermas démontre ainsi le potentiel d’une citoyenneté européenne fondée sur un «patriotisme constitutionnel » (1992). Dans la lignée des écrits habermasiens, la question de l’identité et de l'appartenance européennes figure au centre des préoccupations de nombreux auteurs : Etienne Balibar (1996), J.H.H Weiler (1997), Richard Bellamy et Alex Warleigh (1998 et 2001), Jo Shaw (2000), Andreas Føllesdal (2001), Percy Lehning (2001) et Philippe C. Schmitter (2001) analysent le potentiel d'une citoyenneté européenne à travers le prisme de l’identité européenne. L’ensemble de ces travaux envisagent la possibilité d’une citoyenneté supranationale fondée sur une conception civique ou « éthico-politique » (Mouffe, 1993) de l'appartenance plutôt qu’une conception ethnique/nationale : «Thus, while nominal citizenship by definition implies identity (...), this would not appear to necessitate ethnic or national identity » (Tambini, 2001 : 210).Un certain nombre d'auteurs se fondent néanmoins sur une définition culturelle ou ethnique de l'identité pour concevoir l'émergence d’une citoyenneté supranationale à travers une définition 
Review of European and Russian Affairs vol. 2 issue 3/2006 @ RERA 2006 all rights reserved

culturelle de l'identité. Richard Falk dresse ainsi le portrait d'un citoyen global homme/femmes d'affaires opérant à l'échelle transnationale et immergé dans une culture globale d'élite; il décrit ainsi l'émergence d’une communauté de citoyens globaux businessmen fréquentant les mêmes hôtels à Tokyo, Londres ou New-York, parlant l'Anglais couramment, partageant les mêmes expériences, symboles et infrastructures, etc. (1994: 134). La croissance des migrations internationales a également vu l'émergence d'une littérature prolifique sur les identités et communautés transnationales générées par ce phénomène et constituant une base fertile pour le développement d’une forme dénationalisée de citoyenneté (Basch et al., 1994, Smith, 1998 ; Portes, 1996 ; Ma Mung, 1999; Fitzgerald, 2000).

Cette lecture de la problématique de la citoyenneté supranationale à travers le prisme de l'appartenance et de l'identité collective s'inscrit directement dans la perspective de plusieurs analyses majeures de la citoyenneté dans le cadre national. Dans les écrits de T.H Marshall, la citoyenneté, en tant que statut composé de droits et de responsabilités, est l'expression légale de l'appartenance pleine et entière à une communauté (1949 : 8). Dans son analyse historique, Rogers Brubaker montre dans la même perspective comment la citoyenneté, à la fois objet et instrument d’inclusion et d'exclusion (1992: 23, 34), est historiquement liée à la formation des États-nations français et allemand : « The citizenry of every modern state is internally inclusive (...). In this respect the modern state is not simply a territorial organization but a membership organization, an association of citizens » (1992: 21).

Ces travaux soulignent l'importance de la dimension collective de la citoyenneté. Une analyse complète de la notion de citoyenneté supranationale ne saurait 
donc en faire abstraction. L’idée de communautés post-Westphaliennes dépassant l’Étatnation ainsi que les travaux sur l’identité européenne mentionnés précédemment offrent dans cette perspective un éclairage fructueux sur les bases collectives d'une éventuelle citoyenneté supranationale. Les travaux contemporains sur la définition de l’appartenance au-delà de l’État s’inscrivent cependant généralement dans une perspective, implicite ou explicite, d'évaluation et de prescription. Andrew Linklater présente ainsi explicitement son ouvrage comme un "projet» pour la construction de «communautés postWestphaliennes » (1998). Dans la sphère européenne, un grand nombre d'analyses européanistes visent à évaluer le potentiel de l'UE pour l'instauration d'une citoyenneté européenne (notamment Preuss et al., 2003 : 12) et proposent dans cette perspective un certain nombre de scénarios ou de principes d’appartenance qui pourraient constituer les bases de cette citoyenneté: dans la lignée du “patriotisme constitutionnel” proposé par Jürgen Habermas (1992), Percy B. Lehning propose une théorie de philosophie politique fondée sur le contrat comme base d’une “citoyenneté européenne démocratique libérale” (2001) et Andreas Føllesdal conçoit la citoyenneté européenne à travers la notion de “confiance mutuelle” entre les individus (2001); Richard Bellamy et Alex Warleigh proposent une “éthique de la participation” fondée sur un “communautarisme cosmopolite” (1998) et Philippe C. Schmitter évalue le potentiel de la citoyenneté européenne à la lumière des six “membership norms” définissant la conception idéaltypique de la citoyenneté selon Rogers Brubaker pour présenter un ensemble de réformes visant à renforcer le sentiment citoyen en Europe par une nouvelle conception de la citoyenneté sociale (Schmitter 2001). Ces analyses présentent ainsi souvent un caractère exclusivement philosophique et prescriptif qui laisse peu de place à une analyse 
sociologique ${ }^{3}$ de ce que sont (et non ce que pourraient être) les liens d'appartenance à la base d'une citoyenneté supranationale dans le contexte contemporain.

La notion de « citoyenneté globale » ou « citoyenneté cosmopolite » figure enfin au centre d'un nombre conséquent de travaux cherchant à imaginer la citoyenneté au-delà de l’État-Nation. Dans un plaidoyer contre le patriotisme, Martha Nussbaum affirme ainsi que seul le développement d'un citoyen cosmopolite, «the person whose primary allegiance is to the community of human beings in the entire world », par l'éducation permettra la réalisation des idéaux moraux de justice et d'égalité dans le monde contemporain (1994: 2). Le projet critique défendu notamment par Linklater revêt également une orientation cosmopolite: "Critical international theory defends the political transformation of political community by advocating dialogic communities which are cosmopolitan in orientation, respectful of cultural differences and committed to reducing social and economic inequalities, nationally and internationally » (1998: 109). Dans l'ensemble de ces travaux, c'est l'impératif moral de solidarité qui justifie le développement d’une citoyenneté supranationale; la citoyenneté est ainsi moins envisagée en termes de droits que de responsabilités à l'égard de l'ensemble de l’humanité (Heater 1990 et 2002 ; Wingenbach 1998). La notion de responsabilité comme fondement à une citoyenneté globale est considérée dans une perspective à la fois morale mais aussi «pragmatique » dans les travaux sur la responsabilité à l'égard de l'humanité en matière d'environnement (Hunter 1992 ; van Steenbergen 1994 ; Urry 2000) ou de solidarité Nord-Sud (Arneil, 2005).

\footnotetext{
${ }^{3}$ Bien qu'Andrew Linklater décrive sa démarche comme une entreprise à la fois « normative, sociologique et praxéologique » (1998: 10), son argument est avant tout fondé sur la présentation d’un « projet » de communautés post-Westphaliennes et de citoyenneté transnationale.
} 
Dans la lignée de la philosophie cosmopolite de Kant (1970), l'invocation des notions de solidarité/responsabilité comme fondement de la citoyenneté offre une perspective intéressante pour la sphère supranationale; elle permet de dépasser la conception traditionnelle d'une citoyenneté nécessairement fondée sur une identité partagée. Les travaux s’inscrivant dans cette perspective ne résistent pourtant pas à deux critiques importantes. En premier lieu, l’idée de solidarité ne suffit pas à la construction d’une citoyenneté supranationale effective ; l'exercice de celle-ci suppose en effet des responsabilités et des droits concrets garantis par des cadres d'action institutionnels effectifs (Ignatieff 1991, 34 ; Archibugi 1995). Or, à l'exception de quelques travaux ${ }^{4}$, ceux-ci sont rarement analysés par les avocats d’une citoyenneté globale/cosmopolite. Ensuite, les travaux autour de la citoyenneté globale ou cosmopolite présentent une perspective explicitement normative. « Avocats de la citoyenneté globale » (Sahle, 2005 : 2), ils insistent sur la nécessité, l'impératif de développer la citoyenneté au-delà de l’État dans le contexte contemporain de fragmentation et globalisation: « (...) It is increasingly unlikely, in the context of rising instances of, and opportunities for, transnational harm, that the civic ideal will be preserved if citizens rely exclusively on national institutions. Under these conditions, the ideals of citizenship clash with the sovereign nation-state in which they were first developed» (Linklater, 1998: 182). Dans une analyse récente, Nigel Dower définit en ces termes la notion de «citoyenneté globale»: «a normative claim about how humans should act, an existential claim about what is the case in the world and an aspirational claim about the future » (2003: 6). Dans une perspective similaire, Richard Falk considère que si l'état actuel de la société

\footnotetext{
${ }^{4}$ On pense ici à l'analyse de David B. Hunter liant citoyenneté globale et droit international de l'environnement.
} 
Review of European and Russian Affairs vol. 2 issue 3/2006 @ RERA 2006 all rights reserved

internationale rend tout argument en faveur de l'existence d'une citoyenneté globale irréaliste et idéaliste, le développement de celle-ci comme projet politique ne saurait être abandonné ; dans cette perspective, il suggère le dépassement d’une conception de la politique comme « art du possible » (« axe de faisabilité » en faveur d'une conception de la politique comme « art de l'impossible » (« axe d’aspiration »)(1994 et 2000) : « Global citizenship of a positive variety implies a utopian confidence in the capacity to exceed realistic horizons, but it is also rooted in the highly pragmatic conviction that what is currently taken to be realistic is not sustainable » (1994: 140). Dans l'ensemble de ces travaux, la citoyenneté est présentée comme un idéal à atteindre ; or l’atteinte de cet idéal dans le contexte contemporain suppose la création d’une citoyenneté transnationale. La réalité présente doit donc être transformée afin d’atteindre cette idéal.

\section{Vers une définition intégrée}

Ainsi, si elle offre dans son ensemble un corpus théorique et analytique fructueux qui permet de mettre en lumière les mutations actuelles de la citoyenneté, la littérature contemporaine sur l'émergence d’une citoyenneté européenne, post/trans/supra-nationale ou globale se caractérise en premier lieu par l'absence de consensus concernant la définition même de l’objet analysé. Statut légal composé de droits et de responsabilités, activité politique, expression d’une identité ou de l'appartenance à une communauté, ou encore expression d’un sentiment de solidarité, la citoyenneté est envisagée à travers des perspectives diverses. Il en résulte une conception sous-spécifiée de la citoyenneté ; la notion subit une sorte « d'étirement conceptuel » (Sartori, 1970) et se voit conférer une élasticité apparemment indéfinie, qui semble progressivement lui ôter toute substance. 
Review of European and Russian Affairs vol. 2 issue 3/2006 @ RERA 2006 all rights reserved

Bosniak souligne ainsi «the risk of producing a concept of citizenship that begins to mean very little since it can so readily mean so much » (2000: 487). Les analyses d'une citoyenneté globale fondée sur une solidarité mondiale se prêtent souvent à cette critique (cf. supra).

Limiter le risque d’ «étirement conceptuel » implique alors de s’interroger sur la définition de l’objet «citoyenneté ». Les travaux présentés ci-dessus proposent différentes définitions du concept. Celles-ci renvoient cependant plus à des dimensions complémentaires de la citoyenneté qu’à des conceptions contradictoires. Il convient d'ailleurs de souligner que plusieurs des auteurs mentionnés ne conçoivent pas la citoyenneté à travers une seule de ses dimensions. C’est souvent dans les termes d'une articulation bi-dimensionnelle que s'articule la réflexion de plusieurs européanistes: l’approche de la citoyenneté européenne privilégiée par Jo Shaw (1998 et 2000), ou encore J.H.H Weiler (1997 et 1999) combine à la fois la question des droits et celle de l' appartenance. La perspective en grande partie légale de Elizabeth Meehan (2000) conduit également l'auteure à penser la problématique de la participation des citoyens européens. Dans une même perspective bi-dimensionnelle, les travaux de Yasemin Soysal (1994) et Rainer Bauböck (1993), centrés sur l'internationalisation des régimes de droits, analysent l’implication de la transnationalisation de la citoyenneté comme statut légal pour la définition de l'appartenance à la communauté nationale. C’est également en termes d'articulation droits/appartenance au sein de l’Union européenne que s'articule la réflexion de plusieurs européanistes tels que Jo Shaw (1998 et 2000) ou Weiler (1997 et 1999). Andrew Linklater (1998), avocat déterminé de la citoyenneté cosmopolite, tente lui de combiner les définitions de la citoyenneté comme élément d'appartenance à une 
communauté politique et comme expression de solidarité dans son projet critique de communautés post-Westphaliennes. Enfin, les arguments en faveur d'une citoyenneté globale environnementale combinent souvent une approche juridique de la citoyenneté et une analyse en termes de responsabilité (notamment Hunter, 1992).

En dépit de ces tentatives, la plupart des études sur l'émergence d’une citoyenneté supranationale sont marquées par une vision partielle du concept, la plupart des auteurs concentrant leurs analyses sur une, tout au plus deux, dimensions de la citoyenneté. Face à cette compréhension fractionnée du phénomène, l'élargissement de la définition de la citoyenneté, le développement d’un cadre théorique qui permettrait de la prendre en compte dans toutes ses dimensions semblent nécessaires (Jenson et Phillips, 1996 ; Wiener, 1997 et 1998 ). Cherchant à dépasser les conceptions uniquement juridiques ou politiques qui caractérisent une grande partie des analyses de la citoyenneté, la définition proposée par Bryan S. Turner s’inscrit clairement dans cette perspective: « [a] set of practices (juridical, political, economic and cultural) which define a person as a competent member of society, and which as a consequence shape the flow of ressources to persons and social groups » (1993 : 2). Jenson et Phillips (1996) et Wiener (1997 et 1998) proposent une conception visant non seulement à élargir la définition de la citoyenneté mais également à l'appréhender dans une perspective permettant de rendre compte de ses transformations: à travers la notion de "citizenship practice“, la citoyenneté est conçue comme une relation dynamique entre le citoyen et l'entité politique. Les auteures se sont alors appuyées sur cette conception pour caractériser la citoyenneté et ses transformations dans le cadre de l’État canadien (Jenson et Phillips 1996), mais également dans le cadre supra-étatique de l’Union européenne (Wiener 1997 
et 1998 ; Jenson 2005). Cette conception de la citoyenneté comme relation entre individus et entité politique favorise une approche large et dynamique qui permet de dépasser la compréhension fractionnée du phénomène caractérisant actuellement la littérature sur l'émergence d’une citoyenneté supranationale.

Si ces auteures proposent une conception plus large de la définition de la citoyenneté que celles traditionnellement utilisées dans la littérature, elles n’évacuent pas cependant la nécessité de spécifier cette relation à la lumière de ses fondements historiques. Ainsi, plusieurs auteurs soulignent le lien qui relie citoyenneté et démocratie (Arneil 2005 ; Tully 2005 ; Falk 1994). Dans cette perspective, la citoyenneté comporte au moins deux principes constitutifs : la présence de droits et l'idée de participation (van Gunsteren 1994 ; Falk 1994 ; Arneil 2005). Selon van Gunsteren, le citoyen est à la fois « gouverneur et gouverné » (1994: 37). La citoyenneté étant une relation dynamique entre des individus et une entité politique, Wiener (1997 et 1998), Jenson et Phillips (1996), et Bellamy (2005) ajoutent à ces deux principes caractéristiques de la citoyenneté un troisième élément : l'expression d’une appartenance à une communauté. « Historical studies reveal that debates often turn on three elements of citizenship. The first elements is rights which establish how the individual is legally related to the polity (...). The second element is access which provides information about the conditions for practicing the relationship between citizen and polity (...). The third element is about belonging to a community» (Wiener, 1998: 24-26). La définition de la citoyenneté comme relation dynamique entre individus et entité politique caractérisée par des droits, des canaux institutionnels de participation et la définition d'une appartenance permet ainsi d’appréhender la citoyenneté dans une perspective à la fois globale et dynamique. 


\section{Un débat polarisé : la question du locus de la citoyenneté}

\section{Une problématique unique}

En dépit de leurs divergences de forme (analytique, normative ou prescriptive) et de contenu (définition de la citoyenneté), l'ensemble des travaux contemporains sur la citoyenneté au-delà de l’État sont orientés vers le même argument : la citoyenneté est de moins en nationale et de plus en plus supranationale. Dans cette perspective, ils s'adressent directement aux partisans d'une conception essentiellement nationale de la citoyenneté pour mettre en évidence sa «dénationalisation » (Bosniak, 2000) progressive. Une problématique unique semble donc monopoliser les travaux mentionnés ci-dessus, celle du locus de la citoyenneté : la citoyenneté est-elle (ou doit-elle être) nationale ou dénationalisée ? Les débats se cristallisent ainsi autour d'une perspective dichotomique opposant le «national» au «post-national» (Roche, 1995: 727) : « Increasingly therefore normative debate is polarized between liberal nationalists and cosmopolitans. Cosmopolitans seek to understand the scope for rights, participation and belonging beyond the nation-state, whereas liberal nationalists defend the national model » (Tambini, 2001: 202).

Près de 15 ans après l'émergence des travaux sur la citoyenneté supranationale, cette polarisation binaire enferme les débats dans un cercle stérile. Le dépassement de cette perspective dichotomique nécessite de repenser ses fondements ontologiques. A cette fin, il convient de remarquer que bien qu'ils défendent des arguments radicalement opposés, la grande majorité des travaux contemporains concernant le locus de la 
citoyenneté sont fondés sur une même conception exclusive de la citoyenneté et une même démarche appréhendant la citoyenneté comme un « donné » objectif.

\section{Dépasser la polarisation}

\section{La citoyenneté : conception exclusive vs. approche multiple}

Qu’ils défendent une conception strictement nationale de la citoyenneté ou au contraire celle d'une citoyenneté au-delà de l’État, une grande partie des travaux récents concernant le locus de la citoyenneté véhiculent l’idée d’une citoyenneté conçue comme une relation exclusive entre le citoyen et l'entité politique privilégiée (État, Union européenne, communauté internationale). Martin Kohli dénonce en ces termes la faiblesse des discours contemporains autour de l'identité européenne: «European identity has (...) taken its inspiration from the political science concept of identity as a sense of belonging to some larger political unit, especially as developped in the analysis of nationalism and national identity. The latter leans essentially towards an essentialist or even primordialist understanding of identity - belonging without reservations » (2000: 117). Dominique Schnapper analyse ainsi dans quelle mesure la citoyenneté européenne pourra ultimement remplacer la citoyenneté nationale des États-membres: «Intellectual belief in abstract principles, such as the respect for human rights, legally constituted states, and European citizenship - can these principles really replace, at least in the forseeable future, political and emotional mobilisation which results from the interiorisation of a certain political tradition and national culture? » (2000:11). Les avocats de la citoyenneté supranationale insistent quant à eux sur la nécessité d’envisager « la » citoyenneté au-delà de l’État-nation: Yasemin Soysal affirme ainsi que «la » citoyenneté est de plus en plus post-nationale (1994 et 1997) ; Linda Bosniak s’interroge 
moins sur l'émergence d’une citoyenneté supranationale que sur la dé-nationalisation de « la » citoyenneté (2000). S’érigeant contre ces arguments, les partisans d’une conception strictement nationale affirment ainsi que « la » citoyenneté ne peut prendre sens que dans un cadre étatique (Miller, 1995 et 1999 ; Himmelfarb, 1996). S’ils proposent des arguments radicalement opposés, ces travaux partagent paradoxalement une conception conventionnelle de la citoyenneté fondée sur le postulat que tous les citoyens doivent reconnaître l'autorité suprême d'un seul pouvoir souverain; le lien individu/entité politique (État ou communauté internationale) est présenté comme un lien exclusif.

Cette conception conventionnelle exclusive, dont découle en grande partie la polarisation stérile caractérisant les débats contemporains sur le locus de la citoyenneté, peut être dépassée par une approche autorisant une conception multiple de la citoyenneté. Certains travaux européanistes offrent en ce sens une piste de réflexion fructueuse. L’idée d’une citoyenneté multiple est notamment présente dans les travaux d'Elizabeth Meehan : considérant le lien entre nationalité et citoyenneté comme une relation historiquement contingente plutôt qu’analytiquement nécessaire (2000 : 4), celle-ci affirme qu’à travers la citoyenneté européenne, c'est une nouvelle forme de citoyenneté qui est en train de voir le jour: cette citoyenneté n’est «ni nationale ni cosmopolite »; c’est une forme « multiple », institutionnalisée à travers une configuration de plus en plus complexe liant institutions de la Communauté européenne, États, associations nationales et transnationales, régions et alliances de régions, etc. (1993: 1). Soulignant le caractère primordialiste de la conception de l’identité traditionnellement adoptée dans les travaux sur l’identité européenne, Martin Kohli propose une nouvelle conceptualisation fondée sur la notion d' «hybridation »: "Accordingly, European identity is not empirically 
Review of European and Russian Affairs vol. 2 issue 3/2006 @ RERA 2006 all rights reserved

opposed to national identity. It can and should be conceived as multi-level or multilayered, comprising global and national (and possibly regional and local attachments as well » (2000: 126). «European identity may be part of an identity mix linking it with national (and possibly other territorial) identities; or it may be part of a specifically hybrid pattern where contradictions remain virulent and situational switches occur» (2000: 131). Dans le champ des relations internationales, Andrew Linklater revendique également une conception multiple de la citoyenneté : s’inspirant notamment du scénario de société internationale « néo-médiévale » de Hedley Bull (1977 : 245-246), Linklater développe l’idée d’une citoyenneté multiple fondée sur des entités politiques multiples : «There would be no requirement that the members of [the] community should share one national identity or recognise the supremacy of a single political authority. Breaking with the classical nation-state, post-Westphalian communities would promote a transnational citizenry with multiple political allegiances and without the need for submission to a central sovereign power» (1998:181). Penser la problématique de la citoyenneté supranationale à la lumière d'une conception multiple de la citoyenneté peut également s’appuyer sur un certain nombre de travaux de la littérature, notamment canadienne, entourant la problématique de la citoyenneté dans un cadre national multiculturel (notamment Kymlicka, 1995; Papillon et Turgeon 2003). « To what extent can the citizenship regime associated with a sovereign state be fragmented or redefined 'from within' by small nations? » s’interrogent ainsi Martin Papillon et Luc Turgeon (2003:315). Afin de répondre à cette question, les auteurs comparent l'émergence et la consolidation de régimes de citoyennetés spécifiques au Québec et en Écosse dans le cadre des États canadien et britannique. Montrant alors comment en Écosse, un régime de 
citoyenneté multi-niveaux où les attributs de la citoyenneté sont partagés entre les deux ordres de gouvernement écossais et britannique a progressivement pris forme et au Québec un régime de citoyenneté québécois distinct s’est progressivement constitué en opposition au régime de citoyenneté fédéral, ils proposent une conception non-exclusive de la citoyenneté. Ces analyses offrent un point d'ancrage fructueux pour dépasser l'orthodoxie conventionnelle d'une citoyenneté unique en montrant comment certains contextes sont mieux caractérisés par la superposition, ou l'enchevêtrement, de plusieurs formes de citoyennetés que par une perspective dichotomique cherchant à déterminer « le » locus de la citoyenneté.

\section{Objectivation vs. construction}

Si la polarisation qui caractérise les débats autour du locus de la citoyenneté repose sur une conception essentiellement exclusive de la citoyenneté qui peut être dépassée à la lumière de la littérature sur les citoyennetés multiples, elle procède également d'une approche ontologique marquée par l’objectivation. Qualifier ou évaluer la réalité contemporaine à la lumière de ce qu'est la citoyenneté : telle est la démarche qui sous-tend la majeure partie des travaux contemporains cherchant à déterminer le locus de la citoyenneté, que leur argument soit en faveur d'une citoyenneté nationale ou d'une citoyenneté post-nationale. La citoyenneté est ici appréhendée comme un «donné », quelque chose de « naturel » (Hindess 2000) aux caractéristiques fixées antérieurement à l'analyse à partir de l'histoire occidentale ou de normes morales. Les caractéristiques de la citoyenneté telle qu'elle s’est développée à un certain moment historique dans le cadre d'un espace géographique donné - l’État-nation occidental - sont réifiés pour en devenir 
les caractéristiques objectives et essentielles. «Very often the idea of supranational citizenship has no meaning other than the displacement to a 'higher' echelon of the very characteristics of national citizenship », note ainsi Etienne Balibar (1996: 356). Bien qu'elles cherchent à démontrer une transformation de la citoyenneté, les analyses autour de la citoyenneté supranationale ou européenne offrent au concept un statut essentiellement statique: la citoyenneté est conçue comme un état dont il convient de déterminer le locus. Dans cette perspective, la citoyenneté est souvent pensée en termes de conditions, de pré-requis. La problématique du lien citoyenneté/démocratie permet clairement d'illustrer ce point : si plusieurs auteurs mettent à juste titre en lumière la relation historique entre citoyenneté et démocratie (cf. supra), une grande partie d'entre eux envisagent cette relation en termes conditionnels : la démocratie apparaît comme un pré-requis à l'établissement de la citoyenneté. Certains affirment ainsi qu'il semble encore difficile de parler pleinement de citoyenneté à l'échelle supranationale dans la mesure où les institutions de la sphère internationale ne présentent pas un caractère démocratique (Falk, 2000 :6). D’autres considèrent au contraire le caractère de plus en plus transnational de la société civile et de plus en plus global de l'espace public comme l'émergence de conditions menant au développement d'une citoyenneté supranationale (notamment Walzer, 1995). Dans une même perspective conditionnelle, Dominique Schnapper, répondant aux travaux de Habermas sur la possibilité de créer une citoyenneté européenne fondée sur une appartenance civique, insiste sur la nécessité d’une identité, d’une culture communes, définis en termes de «sentiments », pour la création d’une citoyenneté européenne (2002 : 11). La question du lien entre l’État et la citoyenneté, problématique centrale des débats contemporains auteur du locus de la citoyenneté, est 
souvent appréhendée en ces termes. Dans la lignée de philosophes tels que Hegel ou Arendt, une branche importante de la théorie politique défend l’idée qu'il est absurde de concevoir la citoyenneté au-delà de l’État-nation car celui-ci reste le seul mode d'organisation politique capable de concrétiser idéal civique (Miller, 1995 et 1999 ; Himmelfarb, $1996: 72$ et 74). La citoyenneté ne peut être envisagée qu’en fusion avec les concepts de souveraineté, nationalité et territorialité ; elle perd toute signification quand elle est détachée de l’État-nation. L’État est présenté comme une condition objective à l'existence de la citoyenneté ; l'utilisation du concept de citoyenneté est conditionnelle à l'existence d'un État.

Au nom de la dimension historique du concept, la citoyenneté est donc, dans les arguments contemporains concernant son locus, appréhendée comme un élément donné nécessitant la présence d’un certain nombre de conditions, de critères de nature structurelle (État, démocratie, identité commune). Or il semble que l'histoire de la citoyenneté offre une tout autre lecture de la relation entre ces variables; celle-ci s’inscrit moins dans une perspective unilatérale et statique de conditionnalité que dans une dynamique bilatérale de construction. Le rapport entre la citoyenneté et l’État est tout d'abord dans cette perspective moins un rapport conditionnel (l’État comme pré-requis à la citoyenneté) mais une relation de constitution mutuelle. De nombreux auteurs en effet souligné le rôle de la citoyenneté dans la construction et la consolidation des institutions étatiques aux $19^{\text {ème }}$ et $20^{\text {ème }}$ siècles (Bendix, 1964; Tilly, 1990; Rokkan, 1975; Brubaker, 1992). " The emergence of the institution of citizenship cannot be understood apart from the formation of the modern state and state system. But the converse is equally true: the formation if the modern state and state system cannot be understood apart from the 
emergence and institutionalization of citizenship. (...) the state constitutes itself, and delimits the field of its personal jurisdiction, by constituting its citizenry » (Brubaker, 1992: 72). La citoyenneté ne saurait donc être appréhendée comme une conséquence de l’État ; elle est un construit qui participe du processus même de sa formation. Cette conception de la citoyenneté est présente dans des travaux plus récents sur la construction nationale. A la lumière des cas irlandais et basques, Charles Tilly souligne le lien entre citoyenneté et souveraineté : « On both sides of the state-led/state-seeking gap, political leaders sought to attach citizenship to sovereignty »(1995b: 233). Dans la même perspective, analysant la définition d'un régime de citoyenneté distinct comme une « troisième voie » des nationalismes québécois et écossais, Martin Papillon et Luc Turgeon écrivent: «For the state, it [citizenship] is an important political tool for regulating the nature of the relationship between the state, markets and communities. For political actors, it is also a powerful tool for making claims against established norms and institutions and for greater recognition, inclusion and political rights, In other words, citizenship is a central aspect of modern political struggles and its definition may well vary considerably (...)» (2003 : 317). La problématique d'une citoyenneté au-delà de l’État-nation devrait donc moins se poser en termes statique - chercher à déterminer l'état de la réalité au regard des critères donnés de la citoyenneté - qu’en termes dynamiques : il s'agit alors d'analyser la construction d'une citoyenneté au-delà de l'État-nation en relation avec les processus d'émergence et de constitution d'entités politiques supranationales (UE, ONU notamment). 


\section{Comprendre la citoyennisation}

\section{Citoyennisation et art de gouverner}

Plusieurs auteurs ont ainsi pensé la citoyenneté comme un dispositif de gouvernementalité, i.e comme instrument du gouvernement dans une perspective de gestion de la population (Rose, 2000; Hindess, 2000 et 2002). Barry Hindess (2000, 2002) ancre cette approche dans une perspective supranationale: «In this respect, citizenship functions as one component of a broader regime of population management: a regime which operates first by dividing humanity into discrete sub-populations, each consisting of the citizens of some particular state, and secondly, by assigning to each state not only the right but also the obligation to manage its own internal affairs » (2002: 130). Il montre ainsi comment la citoyenneté est un instrument de gestion au sein d'un régime supranational de gouvernement et en tant que tel est constitutive du système interétatique et de l’État. Nikolas Rose explique comment les dispositifs de gouvernementalité contemporains consistent à renforcer les capacités des individus, dans le sens d'une plus grande civilité notamment : « contemporary strategies for the government of conduct, far from seeking to crush hand eliminate the capacities for action of those persons and forces they act upon, on the contrary seek to foster and shape such capacities so that they are enacted in ways that are broadly consistent with particular objectives such as order, civility, health or enterprise » (2000: 323).

Ces analyses s'inspirent explicitement de l'analyse foucaldienne de la construction de l’État. Selon Michel Foucault, l'élément central du processus de la formation de l'entité étatique renvoie moins à la problématique de sa souveraineté qu’à 
celle de sa «gouvernementalisation »: «Et il est vraisemblable que si l’État existe tel qu'il existe maintenant, c’est grâce, précisément, à cette gouvernementalité qui est à la fois extérieure et intérieure à l’État, puisque ce sont les tactiques de gouvernement qui, à chaque instant, permettent de définir ce qui doit relever de l'État et ce qui ne doit pas en relever, ce qui est public et ce qui est privé, ce qui est étatique et ce qui est non-étatique, Donc, si vous voulez, l’État dans sa survie et l'État dans ses limites ne doivent se comprendre qu’à partir des tactiques générales de la gouvernementalité » (Foucault, 2004 :112-113). (2004:109). Selon Foucault, c'est non pas dans le rapport à un « territoire » mais à une «population » que s'exerce le gouvernement et par conséquent se crée une entité politique (2004 :100). Il montre alors clairement que le gouvernement, et par conséquent le processus de formation de l'entité étatique, s’accompagne du développement d’un «art de gouverner », « de tactiques et de techniques » spécifiques pour agir sur la population : « Naissance d'un art ou, en tout cas, de tactiques et de techniques absolument nouvelles » (2004 : 109).

Adoptant une approche différente, d'autres auteurs ont également mis en évidence l'importance de la définition d'un art de gouverner rationnel dans la construction de l’État et le rôle de la citoyenneté dans ce processus. C'est à travers la création d'une structure organisationnelle répondant aux normes de rationalisation institutionnalisées au niveau mondial que George M. Thomas, John W. Meyer, Franciso O. Ramirez et John Boli, s’inspirant dans leur analyse historique structurelle des travaux de Max Weber, expliquent la constitution de l’État ; John Boli met alors explicitement en évidence le rôle de la citoyenneté dans la mise en place de cet art de gouverner rationnel : « Citizen rights are 'incorporative', serving not so much to strengthen the possibility of individual 
choice as to expand state jurisdiction over the lives of citizens bringing individuals fully into the arena of state action and control » $(1987: 134)$.

\section{La citoyennisation, un processus politique : le rôle des acteurs}

Si les travaux mentionnés ci-dessus permettent de mettre en évidence le rôle des structures dans la construction d'une entité politique et la constitution de la citoyenneté, comprendre la citoyennisation comme processus de construction implique de considérer aussi le rôle des acteurs. Il s'agit de prendre en compte la dimension politique de la citoyenneté, son caractère contesté, et par conséquent ne mettre en exergue le rôle des acteurs dans sa construction. Instrument de gouvernance, la citoyenneté est un enjeu de pouvoir et de ressources, et par conséquent un objet de lutte mobilisé et façonné par des acteurs particuliers dans un contexte particulier. Sa construction est alors le fruit de négociations, d'un échange entre différents acteurs politiques et sociaux. Définissant la subordination de l'intérêt privé à l'intérêt public et de la décision privée à la décision publique comme la condition sine qua non de toute communauté politique, Reinhard Bendix affirme ainsi : «(...) the members of a political community consent to that subordination in an exchange for certain public rights, While governments vary greatly with regard to the subordination they demand and the rights they acknowledge, the term ' political community' may be applied wherever the relations between rulers and ruled involve shared understandings concerning this exchange and hence are based in some measure on agreement » (1964 : 19). L’idée d’échange et de négociations au coeur de la citoyenneté est encore plus explicite dans l'analyse de Charles Tilly. L’auteur montre ainsi comment, dans le cadre du processus de formation de l'État, la citoyenneté a 
émergé comme produit de négociations entre les différents groupes de la société et les autorités en quête ressources pour la conduite de la guerre. Il conclut ainsi: «The core of what we now call 'citizenship’, indeed, consists of multiple bargains hammered out by rulers and ruled in the course of their struggles over the means of state action, especially the making of war» (1990: 102).

La conception d'une citoyenneté à la fois instrument de gouvernance et objet de contestation est également présente dans des travaux plus récents sur la construction nationale. S’appuyant sur les cas irlandais et basques, Charles Tilly montre combien la citoyenneté est à la fois un outil politique et un objet de lutte (1995b: 233). Dans la même perspective, analysant la définition d'un régime de citoyenneté distinct comme une « troisième voie » des nationalismes québécois et écossais, Martin Papillon et Luc Turgeon écrivent: «For the state, it [citizenship] is an important political tool for regulating the nature of the relationship between the state, markets and communities. For political actors, it is also a powerful tool for making claims against established norms and institutions and for greater recognition, inclusion and political rights, In other words, citizenship is a central aspect of modern political struggles and its definition may well vary considerably (...)» (2003: 317).

À la fois instrument de gouvernance et objet de contestation, la citoyenneté doit être appréhendée non pas comme un « donné » universel et atemporel mais comme un « construit » dont la signification est « le produit d'un processus relationnel, culturel, historique et contingent » (Wiener, $1998: 11$ ). C'est dans ces termes que la question de 
la citoyenneté au-delà de l’État-nation, dans une perspective européenne comme dans une perspective internationale, mérite d’être posée : l'approche devient alors moins « substantialiste » (Tambini 2001 : 207) et plus constructiviste. La problématique de l'émergence d'une citoyenneté au-delà de l'État-nation est alors non plus envisagée à travers la caractérisation de l'état de la citoyenneté (nationale vs. supranationale) mais l'analyse de la citoyennisation comme politique au sein du processus de constitution d'entités politiques de gouvernance à l'échelle supranationale. Dans cette perspective, il convient moins de s'interroger sur l'existence des structures conditionnelles de l’établissement de la citoyenneté au-delà de l'État que sur les acteurs, les entrepreneurs politiques contribuant à sa construction et sa définition. 


\section{Bibliographie}

Anheier, Helmut, Marlies Glasius et Mary Kaldor (dir). 2001: Global Civil Society Yearbook 2001. Oxford: Oxford University Press.

Archibugi, Daniele. 1995. «Immanuel Kant, Cosmopolitan Law and Peace». European Journal of International Relations 1, 429-456.

Ardant, Gabriel. 1975. «Financial Policy and Economic Infrastructrure of Modern States and Nations». Dans The Formation of National States in Western Europe, dir. Charles Tilly. Princeton, NJ: Princeton university Press.

Arneil, Barbara. 2005. «Global Citizenship and Empire». Communication présentée à l'atelier The Meaning of Global Citizenship, 9-10 septembre, Liu Centre for Global Issues, University of British Columbia.

Balibar, Etienne. 1996. «Is European Citizenship Possible? ». Public Culture 8:355-376.

Basch, Linda G., Nina Glick Schiller, et Cristina Szanton Blanc. 1994. Nations unbound: transnational projects, postcolonial predicaments, and deterritorialized nationstates. Luxembourg: Gordon and Breach.

Bauböck, Rainer. 1994. Transnational citizenship: membership and rights in international migration. Aldershot, Hants, England; Brookfield, Vermont: E. Elgar.

Bellamy, Richard. 2005. «The Values of Citizenship: Belonging, Rights and Participation». Dans Values in the Constitution of Europe, dir. Miriam Aziz et Susan Millns. Aldershot: Dartmouth.

Bellamy, Richard et Alex Warleigh, dir. 2001. Citizenship and Governance in the European Union. Londres, New York: Continuum.

Bellamy, Richard et Alex Warleigh. 1998. « From an Ethics of Integration to an Ethics of Participation - Citizenship and the Future of the European Union». Millennium: A Journal of International Studies 27: 447-470.

Bendix, Reinhard. 1964. Nation-building and citizenship: studies of our changing social order. New York: Wiley.

Bosniak, Linda. 2000. «Citizenship Denationalized». Indiana Journal of Global Legal Studies 7:pp. 447-509.

Brubaker, William Rogers. 1992. Citizenship and nationhood in France and Germany. Cambridge, Mass.: Harvard University Press. 
Review of European and Russian Affairs vol. 2 issue 3/2006 @ RERA 2006 all rights reserved

Bull, H. 1977. The Anarchical Society: A Study of Order in World Politics. Londres: Macmillan.

Dower, Nigel. 2003. An Introduction to Global Citizenship. Edinburgh University Press.

Falk, Richard. 1994. «The Making of Global Citizenship». Dans The Condition of Citizenship, dir. Barry van Steenbergen. Londres: Sage

Falk, Richard. 2000. «The Decline of Citizenship in an Era of Globalization». Citizenship Studies 4 (1): 5-17.

Fitzgerald, David. 2000. Negotiating Extra-Territorial Citizenship: Mexican Migration and the Transnational Politics of Community. Monograph series no. 2. La Jolla: Center for Comparative Immigration Studies, University of California San Diego.

Føllesdal, Andreas. 2001. « Union Citizenship: Unpacking the Beast of Burden ». Law and Philosophy 20: 313-343.

Foucault, Michel. 2004. «Leçon du 1er février 1978». Dans Sécurité, Territoire, Population. Cours au Collège de France. 1977-1978, dir. M. Foucault. Paris: Gallimard, Seuil.

Habermas, Jürgen. 1992. « Citizenship and National Identity: Some Reflections on the Future of Europe ». Praxis International 12 (1): 1-19.

Habermas, Jürgen.1990. Moral Consciousness and Communicative Action. Cambridge: Polity Press.

Habermas, Jürgen.1989. The Theory of Communicative Action. Cambridge: Polity Press.

Heater, Derek. 2002. World Citizenship: Cosmopolitan Thinking and its opponents. Londres: Continuum

Heater, Derek. 1990. Citizenship: The Civic Ideal in World History, Politics and Education. Londres: Longman.

Himmelfarb, Gertrude. 1996. «The Illusions of Cosmopolitanism». Dans For Love of Country: Debating the Limits of Patriotism, dir. Joshua Cohen et Martha Nussbaum. Boston, MA: Beacon.

Hindess, Barry. 2002. «Neoliberal Citizenship». Citizenship Studies 6 (2): 127-143.

Hindess, Barry. 2000. «Citizenship in the International Management of Populations». American Behavioural Scientist 43 (9):1486-1497. 
Hunter, David B. 1992. «Toward Global Citizenship in International Environment Law». Willamette Law Review 28 (3): 547-563.

Ignatieff, M. 1991. «Citizenship and Moral Narcissism ». Dans Citizenship, dir. G. Andrews. Londres: Lawrence and Wishart.

Jenson, Jane. et Susan D Phillips. 1996. «Regime Shift : New Citizenship Practices in Canada ». International Journal of Canadian Studies 14 (Fall): 111-136.

Jenson, Jane. 2005. « The European Union's citizenship regime. Political and social rights in the era of new governance ». Note de recherche présentée pour l'atelier Contested Meanings: Democratic Practice and Principles across Cultural Boundaries, Queen’s University, Belfast, 22-23 septembre.

Kant, I. 1970. « Perpetual Peace ». Dans The Theory of International Relations: Selected Texts from Gentili to Treitschke, dir. Murray Greensmith Forsyth, H. M. A. Keens-Soper, et Peter Savigear. Londres: Allen \& Unwin.

Kohli, Martin. 2000. «The Battlegrounds of European identity». European Societies 2 (2):113-137.

Kymlicka, Will et Wayne Norman. 1994. «Return of the Citizen: A Survey of Recent Work on Citizenship Theory». Ethics 104:352-381.

Lehning, Percy B. 2001. « European Citizenship: Towards a European Identity? ». Law and Philosophy 20: 239-282.

Linklater, Andrew. 1998. The Transformation of Political Community. Columbia: University of South Carolina Press.

Linklater, Andrew. 2001. «Globalization and the transformation of political community». Dans The globalization of world politics: an introduction to international relations, dir. J. Baylis et S. Smith. Oxford : New York: Oxford University Press.

Ma Mung, Emmanuel. 1999. «La dispersion comme ressource». Cultures \& Conflits 3334: 89-103.

Magnusson, Warren. 1996. The Search for Political Space: Globalization, Social Movements, and the Urban Political Experience. Toronto: University of Toronto Press.

Marshall, Thomas Humphrey. 1963. Sociology at the crossroads : and other essays, Heinemann books on sociology. Toronto: Heinemann.

Meehan, Elizabeth M. 1993. Citizenship and the European Community. Londres ; 
Newbury Park, Calif.: Sage Publications.

Meehan, Elizabeth M. 2000. Citizenship and the European Union. Center for European Integration Studies, Rheinische Friedrich Wilhelms-Universität Bonn, Discussion Paper C 63.

Miller, David. 1995. On Nationality. Oxford: Oxford University Press.

Miller, David. 1999. «Bounded Citizenship». Dans Cosmopolitan Citizenship, dir. Kimberly Hutchnigs et Dann Reuther. Londres: Macmillan Press.

Mouffe, Chantal .1993. The Return of the Political. Londres: Verso.

Nussbaum, Martha. 1994. «Patriotism and Cosmopolitanism». The Boston Review.

Papillon, Martin et Luc Turgeon. 2003. «Nationalism's Third Way? Comparing the Emergence of Citizenship Regimes in Quebec and Scotland». Dans The Conditions of Diversity in Multinational Democracies, dir. A.-G. Gagnon, Montserrat Guibernau et François Rocher. Montréal: Institute for Research on Public Policy.

Portes, Alejandro. 1996. «Global Villagers: The Rise of Transnational Communities». American Prospect 25: 74-77.

Preuss, Ulrich K., Michelle Everson, Mathias Koenig-Archibugi, et Edwige Lefebvre. 2003. «Traditions of Citizenship in the European Union». Citizenship Studies 7 (1):3-13.

Roche, Maurice. 1995. «Citizenship and Modernity». The British Journal of Sociology 46 (4):715-733.

Rokkan, Stein. 1975. «Dimensions of State Formation and Nation-building: A Possible Paradigm for Research on Variations within Europe». Dans The Formation of National States in Western Europe, dir. Charles Tilly. Princeton, NJ: Princeton University Press.

Rose, Nikolas. 2000. «Government and Control». The British Journal of Criminology 40:321-339.

Rotbalt, Joseph (dir.). 1997. World Citizenship: Allegiance to Humanity. Londres: Macmillan Press.

Sahle, Eunice N. 2005. « Global Citizenship and Transnational Civil Society: Theory and Practice». Communication présentée à l'atelier The Meaning of Global Citizenship, 9-10 septembre, Liu Centre for Global Issues, University of British Columbia. 
Sartori, Giovanni. «Concept Misformation in Comparative Politics». American Political Science Review 64 (4): 1033-1053.

Schmitter, Philippe C. 2001. " The scope of citizenship in a democratized European Union: from economic to political to social and cultural? ». Dans European Citizenship - National Legacies and Transnational Projects, dir Klaus Eder et Bernhard Giesen. New York: Oxford University Press, 86-121.

Schnapper, Dominique. 2002. «Citizenship and national identity in Europe». Nations and Nationalism 8 (1):1-14.

Schnapper, Dominique, et Christian Bachelier. 2000. Qu'est-ce que la citoyenneté?, Collection Folio/actuel ; 75. Paris: Gallimard.

Shaw, Jo. 1998. «The interpretation of European Union Citizenship». The Modern Law Review 67 (3): 293-317.

Shaw, Jo. 2000. «The problem of membership in EU citizenship». Dans The European Union and its Order, dir. Z. Bankowski et A. Scott. Oxford: Blackwell.

Smith, Robert C. 1998. «Transnational Localities: Community, Technology and the Politics of Membership Within the Context of Mexico and U.S. Migration». Dans Transnationalism from Below, dir. Michael Peter Smith et Luis Eduardo Guarnizo. New Brunswick, NJ: Transaction Publishers.

Soysal, Yasemin. 1994. Limits of Citizenship. Migrants and Postnational Membership in Europe. Chicago, Londres: The University of Chicago.

Spiro, Peter J. 1999. «The Citizenship Dilemma». Stanford Law Review 51 (3) : 597-639.

Spiro, Peter J. 1997. «Dual Nationality and the Meaning of Citizenship ». Emory Law Journal 46 (4): 1412-1485.

Tambini, Damian. 2001. «Post-national citizenship». Ethnic and Racial Studies 24 (2):195-217.

Tehranian, Majid. 2002. «Democratizing Governance». Dans Democratizing global governance, dir. Esref Aksu et Joseph A. Camilleri. Houndmills England; New York: Palgrave.

Thomas, George, John W. Meyer, Francisco Ramirez et John Boli. . 1987. Institutional structure: constituting state, society, and the individual. Newbury Park, California: Sage Publications. 
Tilly, Charles. 1995a. «Citizenship, Identity and Social History». International Review of Social History 40 (Supplement 3):1-17.

Tilly, Charles. 1995b. «The Emergence of Citizenship in France and Elsewhere». International Review of Social History 40 (Supplement 3):223-236.

Tilly, Charles. 1990. Coercion, capital, and European states, AD 990-1990. Cambridge, Mass.: B. Blackwell.

Trubek, Louise et David Trubek. 2005. « Hard and Soft Law in the Construction of Social Europe : the Role of the OMC ». European Law Journal 11 (3).

Tully, James. 2005. «Two Meanings of Global Citizenship: Modern and Diverse». Communication présentée à l'atelier The Meaning of Global Citizenship, 9-10 septembre, Liu Centre for Global Issues, University of British Columbia.

Turner, Bryan S. (dir). 1993. " Contemporary Problems in the Theory of Citizenship ». In Citizenship and Social Theory, dir. Bryan S. Turner. Londres; Newbury Park, CA: SAGE Publications.

Urry, John. 2000. «Global flows and global citizenship». Dans Democracy, Citizenship and the Global City, dir. Engin F. Isin. Londres: Routledge.

Van Steenbergen, Bart. 1994. «Towards a Global Ecological Citizen». Dans The Condition of Citizenship, dir. Barry van Steenbergen. Londres: Sage.

Van Gunsteren, Herman. 1994. «Four Conceptions of Citizenship». Dans The Condition of Citizenship, dir. Barry van Steenbergen. Londres: Sage.

Walzer, Michael (dir.). 1995. Toward a Global Civil Society. Berghahn Books : New York, Oxford.

Wapner, Paul. 1995. «Politics Beyond the State: Environmental Activism and World Civic Politics». World Politics 47: 311-340.

Weiler, J.H.H. 1997. «To be a European citizen - Eros and civilization». Journal of European Public Policy 4 (4):495-519.

Wiener, Antje. 1997. «Making Sense of the New Geography of Citizenship: fragmented Citizenship in the European Union». Theory and Society 26 (4 (Special Issue on Recasting Citizenship)):529-560.

Wiener, Antje. 1998. 'European Citizenship Practice'. Building Institutions of a NonState. Boulder, Oxford: Westview Press. 
Review of European and Russian Affairs vol. 2 issue 3/2006 (C) RERA 2006 all rights reserved

Wingenbach, Ed. 1998. «Justice After Liberalism: Democracy and Global Citizenship”, Dans Citizenship After Liberalism, dir. Karen Slawner et Mark E. Denham. NewYork: Peter Lang. 